\title{
Observer-Based Perturbation Extremum Seeking Control with Input Constraints for Direct-Contact Membrane Distillation Process
}

\section{Fadi Eleiwi \& Taous Meriem Laleg-Kirati}

To cite this article: Fadi Eleiwi \& Taous Meriem Laleg-Kirati (2017): Observer-Based Perturbation Extremum Seeking Control with Input Constraints for Direct-Contact Membrane Distillation Process, International Journal of Control, DOI: 10.1080/00207179.2017.1314551

To link to this article: http://dx.doi.org/10.1080/00207179.2017.1314551

Accepted author version posted online: 08

May 2017.

Submit your article to this journal $\pi$

III Article views: 8

Q View related articles $\sqsubset$

View Crossmark data $\nearrow$ 
To appear in the International Journal of Control

Vol. 00, No. 00, Month 20XX, 1-21

Publisher: Taylor \& Francis

Journal: International Journal of Control

DOI: https://doi.org/10.1080/00207179.2017.1314551

\title{
Observer-Based Perturbation Extremum Seeking Control with Input Constraints for Direct-Contact Membrane Distillation Process
}

\author{
Fadi Eleiwi $^{\mathrm{a}}$ and Taous Meriem Laleg-Kirati ${ }^{\mathrm{a} *}$ \\ ${ }^{a}$ Computer, Electrical and Mathematical Science and Engineering Division, King Abdullah University of \\ Science and Technology (KAUST), Thuwal 23955, KSA.
}

(Received 00 Month 20XX; accepted 00 Month 20XX)

\begin{abstract}
An Observer-based Perturbation Extremum Seeking Control (PESC) is proposed for a Direct-Contact Membrane Distillation (DCMD) process. The process is described with a dynamic model that is based on a 2D Advection-Diffusion Equation (ADE) model which has pump flow rates as process inputs. The objective of the controller is to optimize the trade-off between the permeate mass flux and the energy consumption by the pumps inside the process. Cases of single and multiple control inputs are considered through the use of only the feed pump flow rate or both the feed and the permeate pump flow rates. A nonlinear Lyapunov-based observer is designed to provide an estimation for the temperature distribution all over the designated domain of the DCMD process. Moreover, control inputs are constrained with an anti-windup technique to be within feasible and physical ranges. Performance of the proposed structure is analyzed, and simulations based on real DCMD process parameters for each control input are provided.
\end{abstract}

Keywords: Perturbation based Extremum Seeking Control, Input constraints, Direct-Contact Membrane Distillation, Nonlinear observer, Dynamic model, Anti-windup technique.

\section{Introduction}

Membrane distillation (MD) is a water desalination process which is based on thermal separation. It utilizes micro-porous membranes as an in-between medium for the feed and the permeate containers $\mathrm{MD}$ allows water vapor and other volatile molecules to pass from the feed side to the permeate side to produce clean water, while preventing other non-volatile impurities from passing through the membrane dry pores L. M. Camacho et al. (2013); Eleiwi, Ghaffour, Alsaadi, Francis, and Laleg-Kirati (2016); Eleiwi and Laleg-Kirati (2014a, 2014b); Francis, Ghaffour, Alsaadi, Nunes, and Amy (2014). The process is driven by pressure gradient, in which it is driven by the temperature difference along the membrane boundary layers.

Of the four common configurations in MD systems, the most popular and readily tested in a laboratory environment is the Direct-Contact Membrane Distillation (DCMD) Khayet (2008). While DCMD has the promising potential to take the lead over a wide range of other existing water desalination methods, such as Multi-Stage Flash (MSF) and Reverse Osmosis (RO) Khayet

*Corresponding author. Email: taousmeriem.laleg@kaust.edu.sa 
(2008); Lawson and Lloyd (1997), some drawbacks realted to its poor in production rate and energy consumption present significant challenges for its industrial commercialization, Khayet and Matsuura ((2011), 249 - 293). These drawbacks make the DCMD process an ideal candidate for control application, especially optimal control techniques, where the objective would be to optimize the trade-off of a maximum production of permeate mass flux while constraining energy which is consumed by the process pumps to the minimum.

Extremum Seeking Control (ESC) is a real time optimal control approach, which dynamically searches for the optimizing inputs using available measurements. Like the Traditional Optimal Control (TOC) strategies Athans and Falb (1966); Kwakernaak (1972) and Model Predictive Control (MPC) E. F. Camacho and Alba (2013); Zeilinger, Jones, and Morari (2011), ESC optimizes a performance function that may depend on the system states, control inputs, initial, and final time values C. Zhang and Ordóñez (2011). Unlike TOC which assumes perfect knowledge about the performance function and MPC which requires heavy computation cost Allgöwer and Zheng (2000); Athans and Falb (1966); E. F. Camacho and Alba (2013); Kahne and Lee (1967); Kwakernaak (1972); Lewis, Vrabie, and Syrmos (2012); Zeilinger et al. (2011), ESC can be used when the performance function is unknown or poorly known. and is designed based on the measurement of the performance function or its derivatives, if available Tan, Li, and Mareels (2013). On the other hand and even if the ESC is a model free approach, the availability of a reliable process model adds more benefits, where it contributes further toward comprehensive control simulations, and provides useful information about the tuning of the involved design parameters before setting real experiments. Therefore, the use of ESC is helpful in model or model-free cases Godoy, Braslavsky, and Agüero (2008); Wang, Krstić, and Bastin (1999); C. Zhang and Ordóñez (2011).

The principal concept of ESC is finding suitable operating set-points that are able to maximize or minimize a desired performance function. This idea had several milestones in its development until it became applicable, especially after the turning point of the stability proof by Krstić et al. in Krstić and Wang (2000). Ever since, many successful applications have been documented and significantly enhanced, to name few: the anti-lock braking system control using ESC Drakunov, Ozguner, Dix, and Ashrafi (1995), the combustion process control for internal combustion engines and gas furnaces with the aid of ESC Åström and Wittenmark (2013), and optimization of the power demand for formation flight Binetti and Ariyur (n.d.). These applications experience more robustness, sensitivity reduction, disturbance rejection with comparable less computation cost with ESC comparing to other optimization methods Soudbakhsh and Annaswamy (2013); Tenne and Goh (2010).

In literature, ESC has had several operating schemes. For example, Krstić et al. Krstić (2000); Krstić and Wang (2000); Wang, Yeung, and Krstić (2000) presented schemes for nonlinear systems, where they are perturbed using an external excitation signal to compute the gradient of the performance function and its direction. Due to its simplicity and good performance, it is considered to be the most popular ESC)scheme, it is called Perturbation based ESC scheme (PESC) Calli, Caarls, Jonker, and Wisse (2012); Krstić (2000); C. Zhang and Ordóñez (2011). Additionally, computing the hessian of the performance function along with gradient help toward applying Newton based optimization technique, and forming a scheme called the Newton based ESC Ghaffari, Krstić, and Nesić (2012). Elsewhere, the excitation can be imposed internally by using sliding mode control, and form the sliding mode based ESC C. Zhang and Ordóñez (2011).

In this work, the main contribution is the adaptation of the PESC by combining it with a nonlinear observer to simultaneously identify and regulate the DCMD process dynamic model. This includes optimizing the trade-off between generating maximum permeate flux, and minimizing energy consumption by the pump flow rates, where either feed pump flow rate is considered to be variable with constant permeate pump flow rate, or both feed and permeate pump flow rates are variable. Accordingly, we use a dynamic model for the DCMD process that is based on the 2D Advection-Diffusion Equation model (2D-ADE) Eleiwi et al. (2016); Eleiwi and Laleg-Kirati (2015, 2014a, 2014b, 2016), where having a reliable process model contributes in tuning the involved 


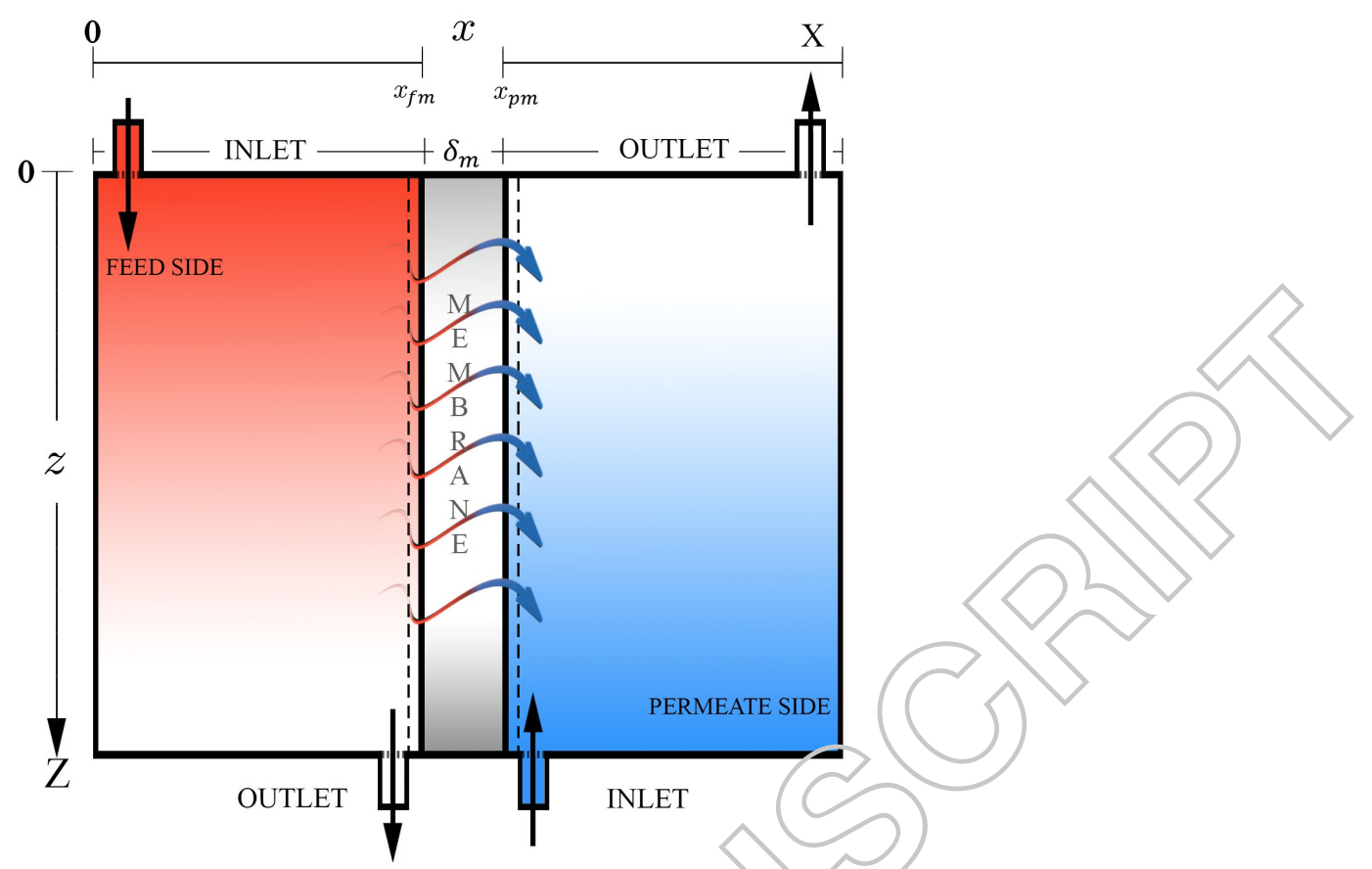

Figure 1. DCMD counter current schematic diagram with the inlets and outlets of hot/cold water flow.

design parameters before setting real experiments. Moreover and following the fact that the process is nonlinear with most of the states are inaccessible, we propose the use of a nonlinear Lyapunovbased observer to provide the PESC with an online and accurate estimate of the process states using the available measurements. Few works in literature have elaborated on the observer based ESC scheme (see Banaszuk, Ariyur, Krstić, and Jacobson (2004); Moase and Manzie (2012)). We consider the physical limitations of the process, therefore we propose the use of anti-windup techniques Galeani and Teel (2006); Grimm, Teel, and Zaccarian (2004); X. Li, Li, Seem, and Li (2012); Tan et al. (2013); Ye and $\mathrm{Hu}$ (2013) to prevent control inputs to wander outside what is physically achievable by the pumps.

This paper is organized as follows: Section 2 introduces the 2D ADE dynamic model for the DCMD process. Section 3 states the problem formulation with a design of PESC and nonlinear observer. Section 4 illustrates simulations for the developed controller for the different control input sets. Finally, section 5 discusses and summarizes the obtained results.

\section{DCMD model}

DCMD process has been best described with dynamic models. In this paper, we consider the DCMD dynamic model presented in Eleiwi et al. (2016); Eleiwi and Laleg-Kirati (2014a).

\subsection{DCMD dynamic mathematical model}

The mathematical model of the DCMD module is presented in Eq. (1), where it represents the reduced model from Eleiwi et al. (2016). The domain of interest is rectangular with finite values for the time, and the space coordinates for each subsystem container as shown in Fig.1.

$$
\frac{\partial T(x, z, t)}{\partial t}+v_{z}^{k} \frac{\partial T(x, z, t)}{\partial z}=\alpha_{x}^{k} \frac{\partial^{2} T(x, z, t)}{\partial x^{2}}
$$


with

$$
k=\{f, p\}, \quad 0<t<\tau_{\text {final }}, \quad x \in\left[0, x_{f m}\right] \cup\left[x_{p m}, X\right], \quad 0<z<Z,
$$

where $k=\{f, p\}$ refers to the feed and the permeate, respectively. $v_{z}^{k}, k=\{f, p\}$ are the feed and permeate pumps flow rates along the $z$ coordinates.

The initial conditions of the model are

$$
T_{f}(x, z, 0)=T_{f_{\text {initial }}}, T_{p}(x, z, 0)=T_{p_{\text {initial }}}
$$

and the associated boundary conditions with the dynamic model (e.g. feed solution) are set to be a Dirichlet condition for the inlet boundary of each subsystem, and Neumann conditions for the rest of the boundaries like Eq. (3).

$$
\begin{cases}T(x, 0, t) & =T_{0_{f}} \text { for } x \in\left[0, x_{m f}\right] \\ T(x, Z, t) & =T_{0_{p}} \text { for } x \in\left[x_{m p}, X\right] \\ \left.\frac{\partial T(x, z, t)}{\partial x}\right|_{x=0} & =0,\left.\quad \frac{\partial T(x, z, t)}{\partial x}\right|_{x=X}=0 \\ \left.\frac{\partial T(x, z, t)}{\partial x}\right|_{x=x_{m f}} & =\left[\mathcal{J}(T) H(T)+\frac{k_{m}}{\delta_{m}} T\left(x_{m p}, z, t\right)\right. \\ & \left.-\frac{k_{m}}{\delta_{m}} T\left(x_{m p}, z, t\right)\right] / k_{f} \\ \left.\frac{\partial T(x, z, t)}{\partial x}\right|_{x=x_{m p}} & =\left[\mathcal{J}(T) H(T)+\frac{k_{m}}{\delta_{m}} T\left(x_{m f}, z, t\right)\right. \\ & \left.-\frac{k_{m}}{\delta_{m}} T\left(x_{m p}, z, t\right)\right] X k_{p},\end{cases}
$$

where $k_{m}$ is the average thermal conductivity of the membrane, $\delta_{m}$ is the membrane thickness, $H(T)$ is the latent heat of evaporation that is responsible of the admissible sensible heat.

$$
H(T)=2500.8-2.36 T+0.0016 T^{2}-0.00006 T^{3} .
$$

$\mathcal{J}(T)$ is the permeate mass flux to be transferred between the feed and the permeate solutions in the process. This mass transport across the membrane in DCMD is generally described by various mass transfer models such as the Kmudsen model or the Poiseuille model or a combination of both Schofield, Fane, and Fell (1987). This transfer is in the form of

$$
\mathcal{J}(T)=C_{m d} \Delta P(T),
$$

where $C_{m d}$ is the membrane distillation coefficient which is function of the membrane properties (pore size, thickness, porosity, and membrane tortuosity), properties of the vapor transported across the membrane (molecular weight and diffusivity), and temperatures. In this paper and without lost of generality, the Knudsen diffusion model (Schofield et al. (1987)), that we adjust by multiplication by a constant $\alpha$, will be considered:

$$
\mathcal{J}(T)=\alpha 1.064 \frac{r \epsilon}{\chi \delta_{m}}\left(\frac{M}{R T_{\text {mean }}}\right)^{\frac{1}{2}} \Delta P(T),
$$

where $r$ is the membrane pore size, $M$ is the molecular weight, $R$ is the gas universal constant, $\chi$ is the membrane tortuosity, and $\epsilon$ is the membrane porosity. $\Delta P(T)$ is the vapor pressure gradient 
between the membrane sides. It is function of the vapor temperature based on Antoine equation and is given by (see L. M. Camacho et al. (2013); Eleiwi and Laleg-Kirati (2014a); Khayet and Matsuura ((2011), $249-293))$ :

$$
P(T)=10^{\left(8.07131-\frac{1730.63}{T+233.426}\right)} .
$$

$T_{\text {mean }}$ is given by

$$
T_{\text {mean }}=\frac{T_{m f}+T_{m p}}{2} .
$$

\section{$2.2 \quad$ Semi-discrete model}

The semi-discrete version of the continuous 2D ADE model is a nonlinear high dimension system of ODEs because of the nonlinearity of the boundary conditions, following the discrete model is given in Eq. (9).

$$
\begin{cases}\dot{\mathbf{T}}(t) & =\mathbf{A}(\mathbf{u}) \mathbf{T}(t)+f(\mathbf{T}(t)) \\ \mathbf{y} & =\mathbf{C T} \\ \mathbf{T}(0) & =\mathbf{T}_{0}\end{cases}
$$

where vector $\mathbf{T} \in \mathbb{R}^{2 n^{2} \times 1}$ is the state vector, which represents the time evolution of the temperature distribution at each grid point inside the feed and the permeate designated domain $\mathbf{T}_{f}, \mathbf{T}_{p}$, respectively.

$$
\mathbf{T}=\left[\begin{array}{c}
\mathbf{T}_{f} \\
\mathbf{T}_{p}
\end{array}\right]
$$

where $\mathbf{T}_{f}, \mathbf{T}_{p} \in \mathbb{R}^{n^{2} \times 1}$ with $n$ the number of discretization points in $x$ and $z$ axes, and $\mathbf{T}_{0}$ is the initial condition vector of the temperature distribution vector. $\mathbf{u} \in \mathbb{R}^{2 \times 1}$ is the input vector which includes the flow rate along the $z$-axis in the feed and permeate solutions, such as:

$$
\mathbf{u}=\left[\begin{array}{l}
u_{f} \\
u_{p}
\end{array}\right]
$$

where

$$
\begin{aligned}
& u_{f}=v_{z}^{f}(z=0), \\
& u_{p}=v_{z}^{p}(z=Z) .
\end{aligned}
$$

Matrix $\mathbf{A}(\mathbf{u}) \in \mathbb{R}^{2 n^{2} \times 2 n^{2}}$ represents the operator matrix that has the dynamics of the feed and the permeate subsystems with their mutual couplings on the boundaries as in Eq. (14), where $i, j$ are the discretization grid indices. $\mathbf{A}(\mathbf{u})$ is a function of the pump flow rates $u_{f}$ and $u_{p}$, in $x, z$ coordinates, for both feed and permeate subsystems, respectively.

$$
\mathbf{A}(\mathbf{u})=\left[\begin{array}{cc}
\mathbf{A}_{f_{i, j}}(\mathbf{u}) & C_{f p} \\
C_{p f} & \mathbf{A}_{p_{i, j}}(\mathbf{u})
\end{array}\right]
$$

where 


$$
\left\{\begin{array}{l}
A_{f_{i, j}\left(u_{f}\right)}=\left(-\frac{u_{f}}{\Delta z}-\frac{2 \alpha_{x}^{f}}{\Delta x^{2}}\right) T_{i, j}+\left(\frac{u_{f}}{\Delta z}\right) T_{i, j-1}+\left(\frac{\alpha_{x}^{f}}{\Delta x^{2}}\right) T_{i-1, j}+\left(\frac{\alpha_{x}^{f}}{\Delta x^{2}}\right) T_{i+1, j}, \\
A_{p_{i, j}\left(u_{p}\right)}=\left(\frac{u_{p}}{\Delta z}-\frac{2 \alpha_{x}^{p}}{\Delta x^{2}}\right) T_{i, j}-\left(\frac{u_{p}}{\Delta z}\right) T_{i, j+1}+\left(\frac{\alpha_{x}^{p}}{\Delta x^{2}}\right) T_{i-1, j}+\left(\frac{\alpha_{x}^{p}}{\Delta x^{2}}\right) T_{i+1, j} . \\
C_{k}=\frac{\alpha_{x}^{k}}{\Delta x^{2}} \frac{\Delta x k_{m}}{\delta_{m} k_{p}}, \quad k=f, p .
\end{array}\right.
$$

where $\Delta x$ and $\Delta z$ are the spatial step sizes in $x, z$ coordinates. $f(\mathbf{T}) \in \mathbb{R}^{2 n^{2} \times 1}$ contains the different boundary conditions of each subsystems, especially the ones associated with the membrane boundary layers. Some of the boundary conditions are nonlinear and functions of the state vector $\mathbf{T}$ as shown in Eq. (3). Finally, we are measuring only the two outlet temperatures in the output vector $\mathbf{y} \in \mathbb{R}^{2 \times 1}$ in Eq.(16)

$$
\mathbf{y}=\left[\begin{array}{c}
T_{\text {fout }} \\
T_{\text {pout }}
\end{array}\right]
$$

with the output matrix $\mathbf{C} \in \mathbb{R}^{2 \times 2 n^{2}}$ in Eq.(17).

$$
\mathbf{C}=\left[\begin{array}{ccc}
\overbrace{0 \cdots 0}^{n^{2}} & \overbrace{10 \cdots 0}^{n-1} & \overbrace{00 \cdots 0}^{n(n-1)+1} \\
0 \cdots \cdots & 0 \cdots \cdots 0 & 10 \cdots 0
\end{array}\right]
$$

\subsection{Model properties}

The DCMD dynamic model is nonlinear, and the source of the nonlinearity results from the nature of the functions $\mathcal{J}(T), H(T)$ and their multiplication in the boundary conditions. However, the present nonlinearity is Lipschitz continuous.

This result can be proved by showing that each of $H(T)$ and $\mathcal{J}(T)$ is separately Lipschitz continuous, and then their multiplication is going to be directly Lipschitz continuous.

For $H(T)$, we follow the fact that it is a polynomial function that is smooth and infinitely differentiable, and therefore it is Lipshitz continuous. Then, we collect all the constants in the expression of $\mathcal{J}(T)$ and write it in a simplified form in Eq. (18).

$$
\mathcal{J}(T)=c_{1}\left(\frac{1}{T}\right)\left(\frac{1}{2}\right)_{10}\left(\frac{-c_{2}}{T}\right) .
$$

where $c_{1}, c_{2}$ are constants, and $T \in[a, b], b>a>0$. Then $\mathcal{J}(T)$ is continuous and differentiable on this interval $[a, b]$, and therefore it is bounded.

$$
\|\mathcal{J}(T)\| \leq \Psi
$$

then there exists a Lipschitz constant $\lambda$ that satisfies Eq. (20)

$$
\left\|f\left(\mathbf{T}_{1}\right)-f\left(\mathbf{T}_{2}\right)\right\| \leq \lambda\left\|\mathbf{T}_{1}-\mathbf{T}_{2}\right\|
$$




\section{Problem formulation}

Improving the performance of the DCMD process is one of the important anticipations for this technique. However, it is not easy, especially with the high rates of energy consumption. Figure 2 shows that for a given inlet temperature, the permeate mass flow rate increases with respect to the feed flow rate and reaches a saturation value at high feed inlet flow rate (figure 1). So it might be interesting to find the optimal flow rate value for a given inlet temperature maximizing the production while minimizing the energy consumption Eleiwi et al. (2016), Karam and Laleg-Kirati (2015).

Figure 2. The permeate flux with respect to the feed flow rate at different feed inlet temperatures

Consequently, we consider the process model in Eq. (9) and compose a performance function as in Eq. (21) that measures the trade-off between the maximization of the permeate flux production and the minimization of the energy consumption. Therefore, optimizing this performance function allows reaching an anticipated optimum trade-off.

$$
\mathbf{J}(\mathbf{T}, \mathbf{u})=\|\mathcal{J}(\mathbf{T})\|-\frac{1}{2} \mu\|\rho \mathbf{u}\|
$$

where $\mathbf{J}$ is a function of the temperature distribution inside the designated process domain through the permeate vapor flux $\mathcal{J}(\mathbf{T})$, and $\mathbf{u}=\left[u_{f}, u_{p}\right]^{T}$ which represents the pump flow rates inside the 
feed and the permeate solutions. $\rho$ is the water density, and it is used to fix the unit consistency for the performance function. $\mu$ is a regularization coefficient, that is used to adjust the weight of each term in the performance function, and sheds more light on certain cases where one term is more dominant over the other. $\|\mathcal{J}(\mathbf{T})\|$ is the Euclidean norm of the permeate vapor flux as in Eq. (6).

Figure 3 shows that the performance function Eq. (21) has a maximum that varies with respect to the inlet temperature. Therefore, we propose in this paper to track the maximum of the cost function by controlling the inlet flow rates.

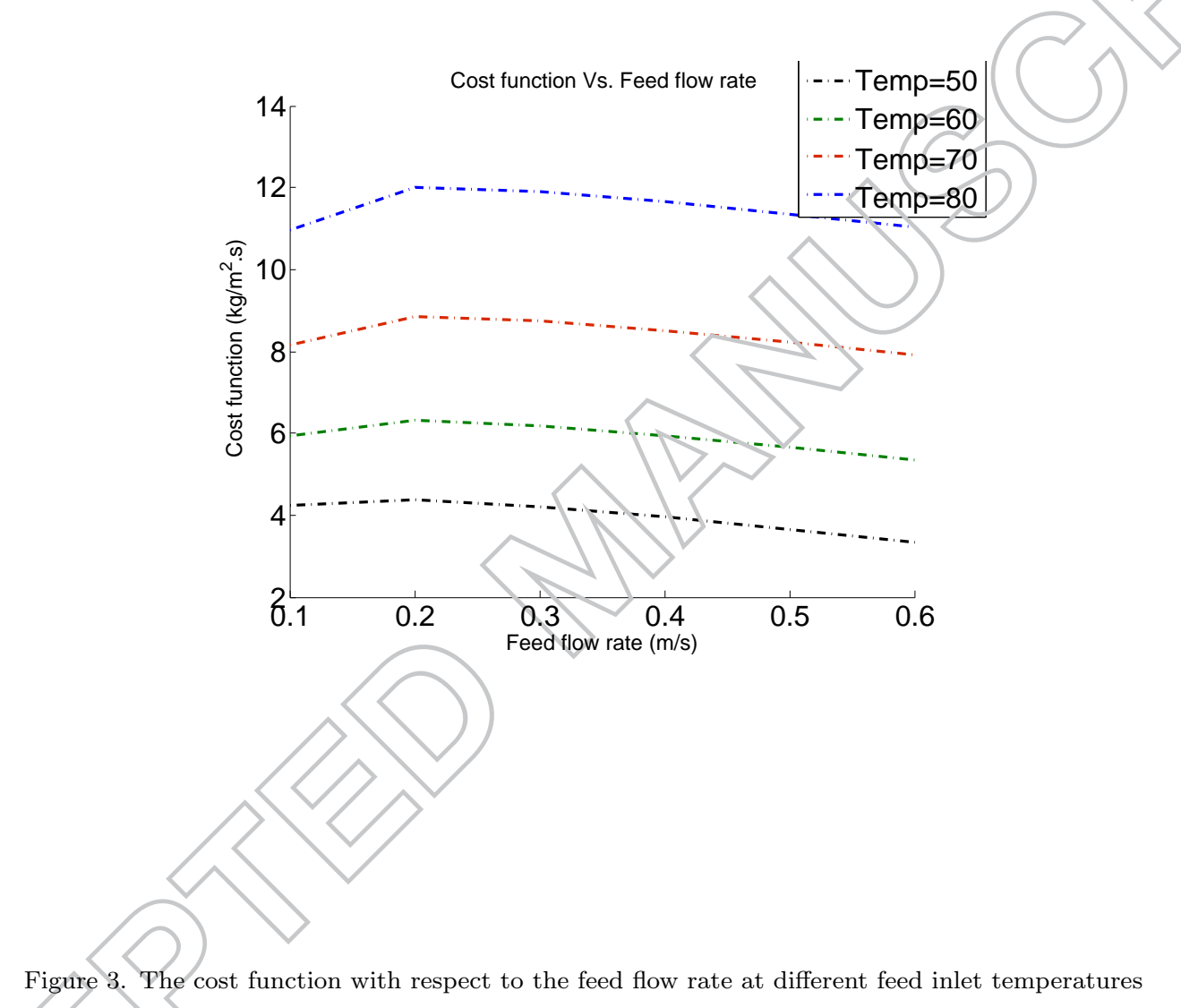

We propose the use of the PESC because of its simplicity and good performance to optimize the performance function in Eq. (21), with no loss of generality to other ESC schemes. In addition, we combine the controller with a nonlinear Lyapunov observer to estimate the temperature distribution inside the designated module domain, and then provide measurements for the performance function. Fig. 4 shows the complete structure of the DCMD dynamic model with the PESC and the nonlinear observer. where $\mathbf{L}$ is the observer gain to be computed in later section, $\widehat{\mathbf{T}}$ and $\widehat{\mathbf{y}}$ are the estimated temperature distribution throughout the process designated domain and the estimated output using the nonlinear observer, respectively.

Next sections highlight the design of PESC and the nonlinear observer. 


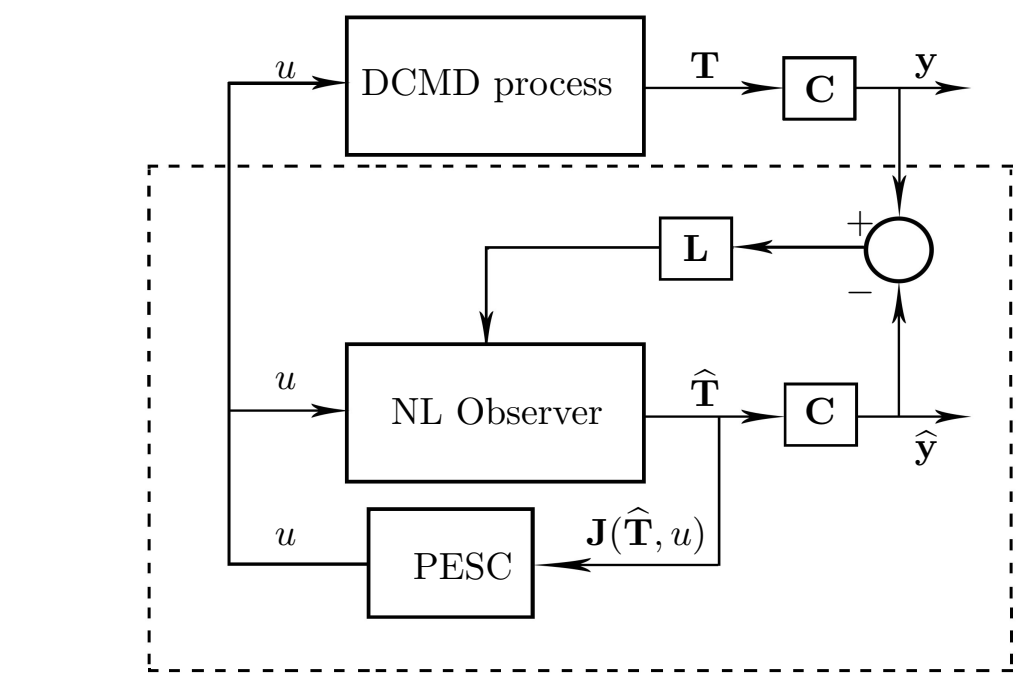

Figure 4. Complete structure of the DCMD model with PESC and nonlinear observer.

\subsection{Extremum Seeking Control}

ESC has advanced properties and advantages in terms of robustness, computation cost effectiveness and disturbance rejection over other optimal control methods Ariyur and Krstić (2003); Liu and Krstić (2012); C. Zhang and Ordóñez (2011). These properties were possible after significant theoretical advancements in the past years, including the proof of the local convergence Ariyur and Krstić (2003); Choi, Krstić, Ariyur, and Lee (2002), slope seeking Ariyur and Krstić (2003); Liu and Krstić (2012), performance improvement and limitations M. Stankovic and Stipanovic (2009); M. S. Stankovic and Stipanovic (2009) to name few.

\subsubsection{Perturbation based ESC}

PESC is a scheme of ESC. It has advantages of performing continuous gradient type optimization with fast adaptation and easy implementation C. Zhang and Ordóñez (2011).

PESC uses a dither signal (usually sine wave) to perturb the performance function and estimate its gradient. Then, it uses the gradient estimation to drive the process to a desired extremum using an integrator and the same dither signal Ariyur and Krstić (2003); Tan et al. (2013); Tan, Nesić, and Mareels (2006). Fig. 5 illustrates the components of a single parameter PESC scheme. A washout filter is one of the components that is used to kill the direct current (DC) component of the output signal, usually this kind of filters is used for signals that will be integrated in time afterwards. Despite the fact that multiplying the output signal with a zero mean dither signal $\sin (\omega t)$ performs the same role, but the washout filter is more effective and does not require the dither frequency $\omega$ to be relatively high Liu and Krstić (2012).

Of interest, ESC has three distinct time-scales Brunton, Fu, and Kutz (2013); Guay, Dhaliwal, and Dochain (2013). Slow time scale to reject any external disturbance, medium time scale which represents the perturbation frequency, and the fast time scale of the internal plant dynamics.

Tuning design parameters in PESC is not a straightforward task and requires a comprehensive knowledge about the time scale of the process, but the availability of the process model is useful and helps in tuning design parameters. Generally literature presents some tuning recommendations (see Ariyur and Krstić (2003); Calli et al. (2012); Deschenes and St-Onge (2013); Liu and Krstić (2012); Moura and Chang (2010); Nesić, Mohammadi, and Manzie (2013); Sharafi, Moase, Shekhar, and Manzie (2013); Tan et al. (2013)). To start, the PESC should view the plant as a static nonlinearity, which means to be slower than the slowest dynamics of the plant and faster than 


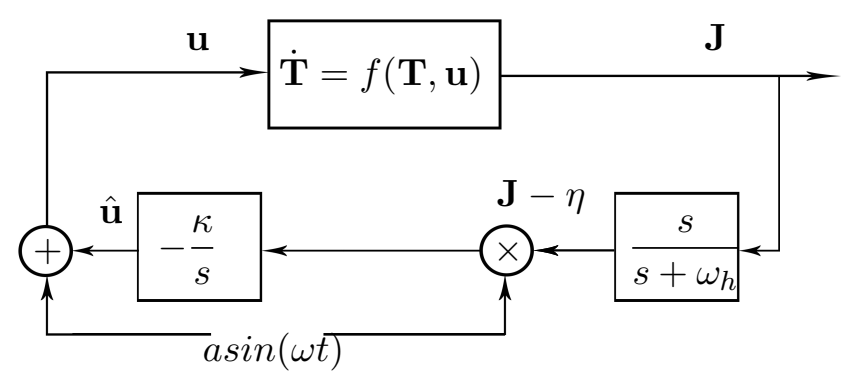

Figure 5. Structure of single parameter PESC.

the disturbance dynamics. Therefore, the frequency of the perturbation signal should be less than dynamics of the plant, such as $\omega<<\min (e i g(A))$ Moura and Chang (2010); Nesić et al. (2013). Moreover, larger controller gain $\kappa$ and perturbation signal amplitude $a$ lead to fast convergence but increase oscillations amplitude and sensitivity disturbance Moura and Chang (2010). Washout cutoff frequency should be less the perturbation signal frequency $\left(\omega_{h}<<\omega\right)$, so-it does not attenuate the perturbation frequency or distort the gradient estimation.

Other ESC designs may include low pass filter to be placed before the integrator, where it is useful to filter out high frequency measurement noise Tan et al. (2006). Additionally, tuning the cut-off frequency of the low pass filter may have improvement in the transient response, but generally it is not a necessary component for the operation of PESC Tan et al. (2013).

Following, we use the PESC to optimize the performance function in Eq. (21) for two control input scenarios. The first is for single control input of the feed pump flow rate, and the second for the multiple control inputs of the feed and the permeate pump flow rates.

\subsubsection{Single parameter PESC}

We consider optimizing the performance function in Eq. (21) to be subject only to the feed pump flow rate $\mathbf{u}=u_{f}$, while the permeate pump flow rate $u_{p}$ is kept constant. It is worth mentioning that in practice, the feed pump flow rate has additional significant influence on the process performance more than the permeate pump flow rate.

The PESC structure in Fig. 5 applies a perturbation signal $\operatorname{asin}(\omega t)$ to a control signal $\widehat{\mathbf{u}}$ that estimates an optimal control $\mathbf{u}^{*}$. The performance function in Eq. (21) is optimized, and necessarily the permeate flux is measured simultaneously through the evaluation of the temperature distribution $\mathbf{T}$ in the $2 \mathrm{D} / \mathrm{ADE}$ model.

At the beginning, the performance function is filtered out by the washout filter. This causes the DC content of the performance function to be eliminated, and as well to be in or out of phase with the perturbation signal asin $(\omega t)$ if $\widehat{\mathbf{u}}$ is less or greater than $u^{*}$, respectively Calli et al. (2012); Moura and Chang (2010). The effect of the washout filter is expressed in Eq. (22).

$$
\left\{\begin{aligned}
\mathbf{J} s & =(\mathbf{J}-\eta)\left(s+\omega_{h}\right) \\
\dot{\eta} & =\omega_{h} \mathbf{J}-\omega_{h} \eta
\end{aligned}\right.
$$

The resultant signal is multiplied with a perturbation signal $\operatorname{asin}(\omega t)$ that has a small amplitude $a$, and provides a signal that has a DC content which is less or greater than zero if $\widehat{\mathbf{u}}$ is greater or less than $\mathbf{u}^{*}$, respectively. Following, an integrator of a gain $\kappa$ extracts the gradient direction of the resultant signal to form the adaptation signal $\widehat{\mathbf{u}}$ Tan et al. (2013).

$$
\dot{\hat{\mathbf{u}}}=a \kappa(\eta-\mathbf{J}) \sin (\omega t),
$$

Later, the adaptation signal $\widehat{u}$ shifts the perturbation signal towards the gradient direction and 
converges to the optimal control, as described in Eq. (24).

$$
\mathbf{u}=\widehat{\mathbf{u}}+\operatorname{asin}(\omega t) .
$$

Stability analysis and convergence to optimal values inside ESC schemes were proved using averaging theorem by Krstić in many studies Ariyur and Krstić (2003); Krstić and Wang (2000); Liu and Krstić (2012), but more elaboration was put in other studies (see Moura and Chang (2010); Nesić (2009); Nesić, Tan, Moase, and Manzie (2010); Tan et al. (2006)).

\subsubsection{Multiple-parameter PESC}

To broaden further the optimization analysis on the DCMD process, we consider optimizing the performance function in Eq. (21) to be subject to both the feed and the permeate pump flow rates as control inputs. This would considerably highlight on the impact of the feed pump flow rate and the permeate pump flow rate on the process production, and the interference between them.

The optimization procedure is similar to the single parameter case, however the multipleparameter PESC considers each control input in a separate loop with perturbation signal that has specific frequency with distinct phase shifts, such as $\omega_{1} \neq \omega_{2}, \phi_{1} \neq \phi_{2}$ (e.g. $\phi_{1}=0$ and $\left.\phi_{2}=\pi / 2\right)$. These phase shifts are introduced to compensate for the delays provided by the plant dynamics and the performance function $\mathbf{J}(\mathbf{T}, \mathbf{u})$ in Eqs. (9), (21), respectively Krstić (2000); Moura and Chang (2010); Nesić et al. (2013); Tan et al. (2013).

The design parameters are set for each input independently, and then the whole process is tuned out to have the optimal behavior Calli et al. (2012). Following equations show the dynamic representation for the feed and the permeate pump flow rates.

$$
\left\{\begin{aligned}
\dot{\hat{\mathbf{u}_{p}}} & =a_{1} \kappa_{1} \sin \left(\omega_{1} t-\phi_{1}\right)\left(\eta_{1}-\mathbf{J}\right), \\
\dot{\overrightarrow{\mathbf{u}_{f}}} & =a_{2} \kappa_{2} \sin \left(\omega_{2} t-\phi_{2}\right)\left(\eta_{2}-\mathbf{J}\right), \\
\dot{\eta_{1}} & =-\omega_{h 1} \eta_{1}+\omega_{h 1} \mathbf{J} . \\
\dot{\eta_{2}} & =-\omega_{h 2} \eta_{2}+\omega_{h 2} \mathbf{J}
\end{aligned}\right.
$$

Afterwards, each control inputs will consist of the adaptation signal in addition to the perturbation signal to converge toward the optimal control.

$$
\left\{\begin{array}{l}
\mathbf{u}_{p}=\widehat{\mathbf{u}_{p}}+a_{1} \sin \left(\omega_{1} t\right), \\
\mathbf{u}_{f}=\widehat{\mathbf{u}_{f}}+a_{2} \sin \left(\omega_{2} t\right) .
\end{array}\right.
$$

Fig. 6 illustrates the diagram of the main components for the multiple-parameter PESC.

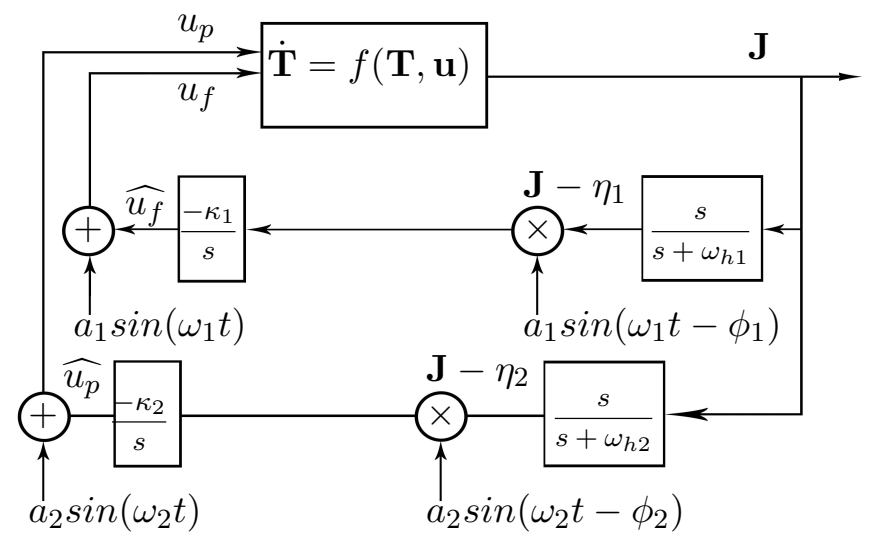

Figure 6. Structure of multiple parameters PESC. 


\subsubsection{Anti-windup PESC}

The presence of an integrator in PESC causes, in some occasions, the control inputs to wander outside their acceptable and feasible domain. This may facilitate the actual inputs to saturate on the actuators (e.g. pumps) domain boundary Tan et al. (2013). This phenomenon is similar to the integrator wind-up in classic control Simmons (1995).

As a solution, we consider an anti-windup technique to overcome input saturation phenomenon. This technique is preferable rather than dealing with the saturation as constant, since the process is dynamic and more likely to have input saturation Åström and Wittenmark (2013); Galeani and Teel (2006); Grimm et al. (2004). Moreover, it allows to impose certain constraints on the control inputs to bound them with physical limits. Technically, anti windup drives the actuator back to the unsaturated mode when input saturation is about to happen by using a penalty-like scheme to prevent or overcome saturation P. Li, Li, and Seem (2009); X. Li et al. (2012); Tan et al. (2013); $\mathrm{Ye}$ and $\mathrm{Hu}$ (2013). Therefore, we combine the anti-windup technique along with the PESC-scheme, and define a saturation function that covers all the scenarios of the control inputs, like in Eq. (27).

$$
\operatorname{sat}\left(\mathbf{u}, \mathbf{u}_{s}\right)=\left\{\begin{array}{cll}
u_{\max } & \text { if } & \mathbf{u} \geq u_{\max } \\
\mathbf{u} & \text { if } & u_{\min } \leq \mathbf{u} \leq u_{\max } \\
u_{\min } & \text { if } & \mathbf{u} \leq u_{\min }
\end{array}\right.
$$

where $\mathbf{u}_{s}=\left[u_{\max }, u_{\min }\right]^{T}$ and $u_{\max }, u_{\min }$ are the upper control input limit and the lower control input limit, respectively. $K_{w}$ is a design parameter that adds more significance to the saturation function weight. The saturation function is placed before the process, and defines an appropriate input $u_{\text {actual }}$ for a feedback. Fig. 7 shows a diagram for a single parameter PESC with anti-windup technique.

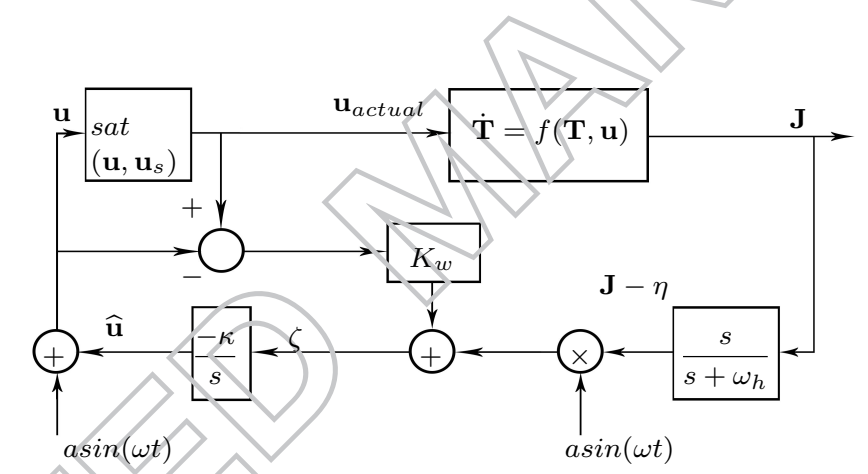

Figure 7. Structure of multiple-parameters PESC with anti-windup technique.

Similarly, the adaptation signal is evaluated, however it includes the effect of the anti-windup. Following equations present the adaptation signal.

$$
\left\{\begin{aligned}
\dot{\hat{\mathbf{u}}} & =a \kappa(\eta-\mathbf{J}) \sin (\omega t) \\
& -\kappa K_{w}\left(\operatorname{sat}\left(\mathbf{u}, \mathbf{u}_{s}\right)-\widehat{\mathbf{u}}-\operatorname{asin}(\omega t)\right) \\
\dot{\eta} & =\omega_{h} \mathbf{J}-\omega_{h} \eta
\end{aligned}\right.
$$

Same procedures are applicable to multiple-parameter PESC, where the anti-windup model is added to each control input channel to prevent input saturation Tan et al. (2013).

\subsection{Observer based PESC}

The PESC requires the performance function measurements to be always available. In practice this is not viable, since the performance function is dependent on some inaccessible temperature states over the process designated domain. On the other hand, the process model is observable, and the 
present nonlinearity is Lipshitz continuous. For these reasons, we propose the use of a nonlinear observer to estimate the process inaccessible states, and provide measurements for the performance function back to the PESC.

\subsubsection{Nonlinear Lyapunov-based observer design}

Nonlinear observers vary in designs depending on the available information about the nature of the system nonlinearity Bestle and Zeitz (1983); Thau (1973); Walcott and Zak (1987); H. Zhang, Chan, Cheung, and Jin (1998). In our case, we have the advantage of a good model for the present nonlinearity, therefore we consider the nonlinear observer presented by Thau et al. in Thau (1973) with the modification of Raghavan et al. in Raghavan and Hedrick (1994) to find an adequate observer gain.

Accordingly, we slightly modified and adapted the work in Raghavan and Hedrick (1994) to the nonlinear model of the DCMD process in Eq. (9), where originally the stability proof was done for a slightly different class of nonlinear system like in Eq. (29).

$$
\dot{T}=\mathbf{A} T+\mathbf{B} u+f(T) .
$$

where $\mathbf{A}$ is the dynamics matrix, $\mathbf{B}$ is the input matrix, $f(T)$ is the nonlinearity vector in the model and $T$ is the state vector.

For the observer design, we consider the nonlinear Lyapunov-based observer in the following form:

$$
\dot{\hat{\mathbf{T}}}(t)=\mathbf{A}(\mathbf{u}) \widehat{\mathbf{T}}(t)+f(\widehat{\mathbf{T}}(t))+\mathbf{L}(\mathbf{y}-\mathbf{C} \widehat{\mathbf{T}}),
$$

where $\widehat{\mathbf{T}} \in \mathbb{R}^{2 n^{2} \times 1}$ is the estimated state vector, and $\mathbf{L}$ is the observer gain matrix.

Then the observer error dynamic equation is given by:

$$
\dot{\widetilde{\mathbf{T}}}=(\mathbf{A}(\mathbf{u})-\mathbf{L} \mathbf{C}) \widetilde{\mathbf{T}}+f(\mathbf{T})-f(\widehat{\mathbf{T}}) .
$$

where $\widetilde{\mathbf{T}}=\mathbf{T}-\widehat{\mathbf{T}}$ is the state estimation error.

Proposition 1 gives sufficient conditions for exponential stability of the state observer error expressed in Eq. (31).

Proposition 1: Assuming $\mathbf{u}$ such that the pair $(\mathbf{A}(\mathbf{u}), \mathbf{C})$ is observable and the nonlinearity is Lipshitz continuous, then there exists an exponentially stable observer of the form Eq. (30) if there exists a symmetric positive definite matrix, $P$, such that the following standard algebraic Ricatti equation is satisfied:

$$
\mathbf{A}(\mathbf{u}) P+P \mathbf{A}(\mathbf{u})^{T}+P R_{1} P+Q_{1}=0
$$

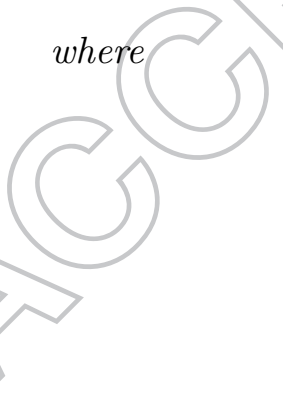

$$
\begin{aligned}
R_{1} & =\left(\gamma^{2} I-\frac{\mathbf{C}^{T} \mathbf{C}}{\epsilon}\right) \\
Q_{1} & =I(\epsilon+1) \\
\epsilon & >0, \quad \epsilon \in \mathbb{R}^{+} .
\end{aligned}
$$


and the observer gain matrix $\mathbf{L}$ is given by:

$$
\mathbf{L}=\frac{P \mathbf{C}^{T}}{2 \epsilon}
$$

We use Lemma 1 to prove Proposition 1.

Lemma 1: Let $\|X\|,\|Y\|$ be the Euclidean norm of vectors $X$ and $Y$. Then, the following inequality holds:

$$
\|X\| .\|Y\| \leq \frac{\left\|X^{2}\right\|}{4}+\left\|Y^{2}\right\|
$$

Proof. let us define for a fixed $\mathbf{u}, \mathbf{A}_{0}(\mathbf{u})=\mathbf{A}(\mathbf{u})-\mathbf{L C}$, and a Lyapunov function $V$ such that in Eq. (38) for the stability, and its derivative in Eq. (39).

$$
\begin{aligned}
V & =\widetilde{\mathbf{T}}^{T} P \widetilde{\mathbf{T}} \\
\dot{V} & =-\widetilde{\mathbf{T}}^{T}\left[\mathbf{A}_{0}(\mathbf{u})^{T} P+P \mathbf{A}_{0}(\mathbf{u})\right] \widetilde{\mathbf{T}}+2 \widetilde{\mathbf{T}}^{T} P[f(\mathbf{T})-f(\widetilde{\mathbf{T}})]
\end{aligned}
$$

where $P \in \mathbb{R}^{2 n^{2} \times 2 n^{2}}$ is any arbitrary symmetric positive definite matrix.)

$$
2 \widetilde{\mathbf{T}}^{T} P[f(\mathbf{T})-f(\widetilde{\mathbf{T}})] \leq 2\|P \widetilde{\mathbf{T}}\| \gamma\|\widetilde{\mathbf{T}}\|
$$

This is valid, since $f(\mathbf{T})$ is Lipschitz, then by using Lemma 1, we define:

$$
\left\{\begin{array}{l}
\|X\|=2 \gamma\|P \widetilde{\mathbf{T}}\| \\
\|Y\|=\|\widetilde{\mathbf{T}}\|
\end{array} .\right.
$$

Then

$$
2 \gamma|| P \widetilde{\mathbf{T}}|||\widetilde{\mathbf{T}}| \mid \leq \gamma^{2} \widetilde{\mathbf{T}}^{T} P P \widetilde{\mathbf{T}}^{T}+\widetilde{\mathbf{T}}^{T} \widetilde{\mathbf{T}}
$$

$$
\vec{K} \leq \widetilde{\mathbf{T}}^{T}\left[\mathbf{A}_{0}(\mathbf{u})^{T} P+P \mathbf{A}_{0}(\mathbf{u})+\gamma^{2} P P+I\right] \widetilde{\mathbf{T}} .
$$

Let

$$
\mathbf{A}_{0}(\mathbf{u})^{T} P+P \mathbf{A}_{0}(\mathbf{u})+\gamma^{2} P P+I=-\epsilon I .
$$

Then

$$
\dot{V} \leq-\epsilon \widetilde{\mathbf{T}}^{T} \widetilde{\mathbf{T}}<0 \quad \text { so } \quad \widetilde{\mathbf{T}} \rightarrow 0 \quad \text { exponentially }
$$

$$
(\mathbf{A}(\mathbf{u})-\mathbf{L C}) P+P(\mathbf{A}(\mathbf{u})-\mathbf{L} \mathbf{C})^{T}+\gamma^{2} P P+I(\epsilon+1)=0
$$

By setting $\mathbf{L}=\frac{P \mathbf{C}^{T}}{2 \epsilon}, R_{1}=\left(\gamma^{2} I-\frac{\mathbf{C}^{T} \mathbf{C}}{\epsilon}\right)$ and $Q_{1}=I(\epsilon+1)$, we obtain a standard algebraic Ricatti equation in Eq. (32) that has a solution if $P$ is symmetric and positive definite matrix. 
Fig. 8 shows the fast convergence of the estimated states using the nonlinear Lyapunov-based observer for different points on the DCMD discretization grid. The numbers in the legends (e.g. $10,20,30$..) represent the points locations along the membrane boundaries. We are more concerned about points along the membrane boundaries because of their significance in driving the process. It shows also how the nonlinear observer responses to any sudden changes in the process. The fast convergence of the observer is guaranteed by taking smaller sampling time Sassano, Carnevale, Astolfi, and Vergata (2011). In the same context, the error analysis shows the exponential convergence of the error to zero as depicted in Fig. 9.

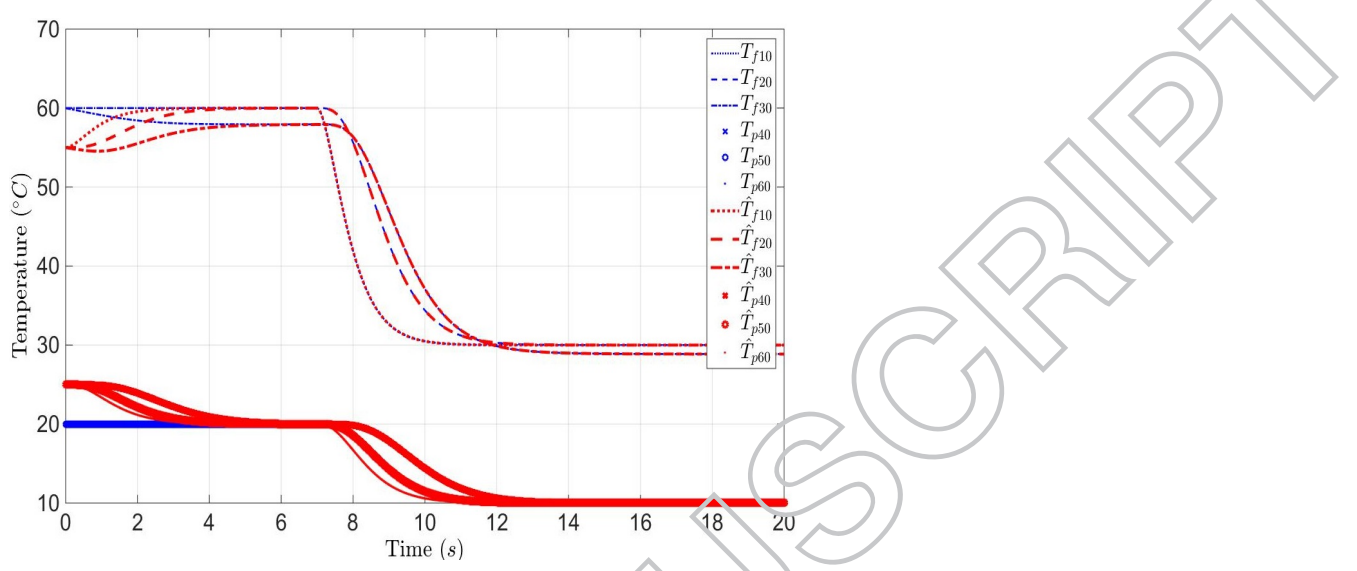

Figure 8. Convergence of the nonlinear observer, where each curve represents a specific location along the membrane boundaries.

\subsubsection{Observer based PESC structure}

Following the design of the nonlinear Lyapunov-based observer, the temperature distribution all along the designated process domain is available at each instant of time. Proceeding further, we can provide measurements of the performance function to the PESC to find optimal control value. This is valid if the observer convergence is fast enough, therefore the stability of the complete structure of the observer-based PESC is vital. For this purpose, the performance function will be expressed as follows:

$$
\mathbf{J}(\hat{\mathbf{T}}, \mathbf{u})=-\|\mathcal{J}(\hat{\mathbf{T}})\|+\frac{1}{2} \mu\|\rho \mathbf{u}\|
$$




\section{Simulations}

In this section, further analysis for the observer based PESC scheme is discussed through numerical simulations over the DCMD process dynamic model in Eq. (9) for each input case. The PESC scheme is equipped with anti-windup technique to prevent input saturation. All fabrication parameters of the employed membrane are realistic, and supplied by the Water Desalination and Reuse Center (WDRC) ${ }^{1}$ at KAUST. These parameters are listed in Table 1.

\begin{tabular}{c|c}
\hline \hline Parameter & Value Membrane \\
\hline \hline material & PTFE $(\mathrm{M} 1)$ \\
\hline Liquid Entry Pressure $(P S I)$ & 15 \\
\hline Thickness $(\mu \mathrm{m})$ & 176 \\
\hline Mean flow pore size $(\mu \mathrm{m})$ & 0.24 \\
\hline First bubble point $(\mu \mathrm{m})$ & 0.42 \\
\hline Porosity $(\%)$ & 70 \\
\hline Contact angle & $140^{\circ} \pm 3^{\circ}$ \\
\hline Mass Transfer Coefficient $\left(\mathrm{Kg} / \mathrm{h} . P a . \mathrm{m}^{2}\right)$ & 0.001 \\
\hline Feed inlet temperature $\left({ }^{\circ} \mathrm{C}\right)$ & 60 \\
\hline Permeate inlet temperature $\left({ }^{\circ} \mathrm{C}\right)$ & 20 \\
\hline
\end{tabular}

Table 1. Characteristics of the employed membrane.

For the single parameter case, we consider the feed pump flow rate $\left(u=u_{f}\right)$ to be the only control input, while keeping the permeate pump flow rate constant at $u_{p}=0.2(\mathrm{~m} / \mathrm{s})$. From practical perspectives, $u_{f}$ has to be bounded with minimum and maximum values to respect pumps physical limitations and energy management, following values are provided by the WDRC in Eq. (48).

$$
0.1(\mathrm{~m} / \mathrm{s}) \leq u_{f} \leq 0.4(\mathrm{~m} / \mathrm{s})
$$

The simulation is depicted in Fig. 10, where it illustrates the permeate flux and the optimized feed pump flow rate $\left(u_{f}\right)$. Noticeably, the control input oscillates in small neighborhood around the optimal value, which is less than its maximum bound. The values of the design parameters are listed in Table 2, and the initial value of the feed flow rate is $0.2 \mathrm{~m} / \mathrm{s}$.

\begin{tabular}{cc|c|c|c}
\hline$\omega_{1}$ & $5 \mathrm{~Hz}$ & $\omega_{2}$ & $4 \mathrm{~Hz}$ \\
\hline$\omega_{h 1}$ & $0.5 \mathrm{~Hz}$ & $\omega_{h 2}$ & $0.5 \mathrm{~Hz}$ \\
\hline$\kappa_{1}$ & 0.1 & $\kappa_{2}$ & 2.1 \\
\hline$a_{1}$ & 0.015 & $a_{2}$ & 0.02 \\
\hline$\phi_{1}$ & $\pi / 2$ & $\phi_{2}$ & 0 \\
\hline
\end{tabular}

Table 2. PESC design parameters.

In different simulation, we introduced a sudden disturbance to the DCMD process to assess the sensitivity as well as the adaptability of the PESC scheme. The disturbance happens by lowering the feed inlet temperature from $60^{\circ} \mathrm{C}$ to $30^{\circ} \mathrm{C}$ at one-third of the full time. Fig. 11 shows the response of the control input to the disturbance. It is important to mention that the permeate flux is generated more significantly at high temperature, and this explains the low generation of permeate flux at $30^{\circ} \mathrm{C}$.

\footnotetext{
${ }^{1}$ Website: https://wdrc.kaust.edu.sa/Pages/Home.aspx
} 

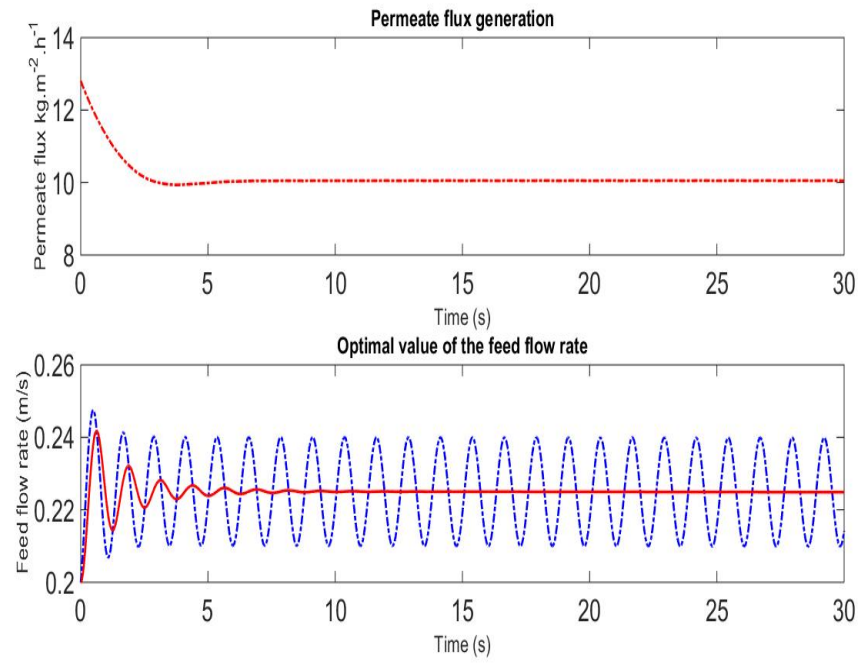

Figure 10. Single parameter PESC with anti-windup technique.

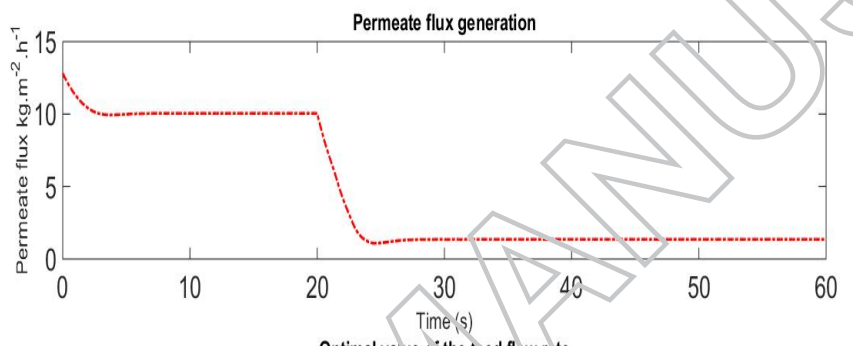

Optimal var ie c the fo od fle w re te

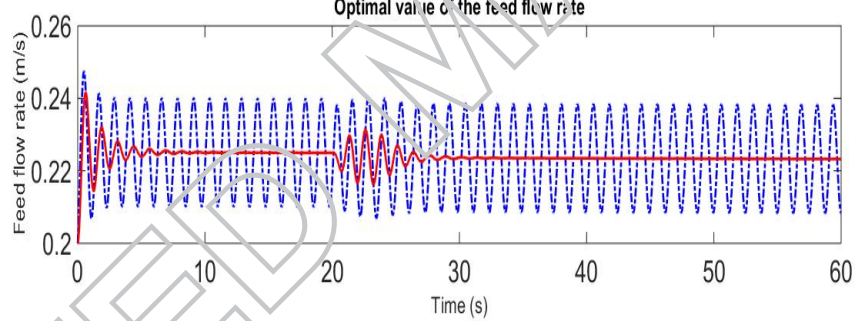

Figure 11. The response of the PESC to sudden disturbance in the process.

For the multiple-parameter case, the performance function is optimized considering the feed and the permeate pump flow rates as control inputs $u=\left[u_{f}, u_{p}\right]^{T}$, with initial values for the feed and permeate flow rates to be $[0.2,-0.2]$, respectively. Fig. 12 demonstrates the time evolution of the permeateflux and the optimized control inputs. It shows also the rapid convergence of the permeate flux to its maximum value, and the saturation of the control inputs.

It is noticeable from the simulations results, that the permeate flux in the multiple-parameter case reaches higher level when comparing to the single parameter case (around $\sim 11.5 \frac{\mathrm{kg}}{\mathrm{m}^{2} . h}$ ), but with higher pump flow rates. This can be justified with the additional degrees of freedom that are provided to the process, by assigning a second control input, where the newly added design parameters can be elaborately tuned, and therefore, contribute toward fast convergence for the performance function. 

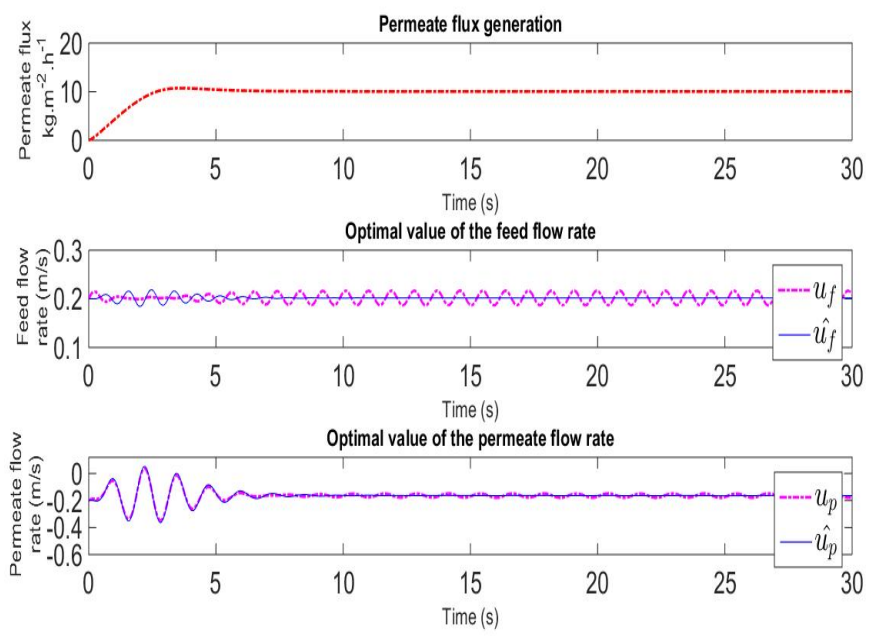

Figure 12. Multiple parameters PESC with anti-windup technique.

\section{Conclusions}

In this work, we propose an observer based PESC for a DCMD process. The process is modeled with a dynamic 2D ADE model. The objective of the controller is to maximize the permeate vapor flux while at the same time to minimize the consumed energy via the feed and permeate pump flow rates. For this purpose, we designed a nonlinear Lyapunov-based observer to provide an estimate for all the temperature distribution among the domain of the process and feed them to the PESC. We consider two sets of control inputs for the PESC scheme: single control input of the feed pump flow rate, and multiple control inputs of both feed and permeate pump flow rates, and provided them with anti-windup technique to prevent actual input saturation. Simulations and analyses for both control input cases are provided. Additionally, simulations show the time evolution of the control inputs and support the feasibility of their energy consumption. Future plans require experimental validation for the simulations to support the results we obtained.

\section{Acknowledgment}

The authors acknowledge that the research reported in this publication was supported by the King Abdullah University of Science and Technology (KAUST) and are grateful for the support provided.

\section{References}

Allgöwer, F., \& Zheng, A. (2000). Nonlinear model predictive control (Vol. 26). Birkhäuser Basel. Ariyur, K. B., \& Krstić, M. (2003). Real-time optimization by extremum-seeking control. WileyBlackwell.

Åström, K., \& Wittenmark, B. (2013). Adaptive control: Second edition. Dover Publications.

Athans, M., \& Falb, P. (1966). Optimal control: an introduction to the theory and its applications. McGraw-Hill. 
Banaszuk, A., Ariyur, K. B., Krstić, M., \& Jacobson, C. A. (2004). An adaptive algorithm for control of combustion instability. Automatica, 40(11), 1965 - 1972.

Bestle, D., \& Zeitz, M. (1983). Canonical form observer design for non-linear time-variable systems. International Journal of Control, 38(2), 419-431. doi:

Binetti, P., \& Ariyur, K. B. (n.d.). Formation flight optimization using extremum seeking feedback. Journal of Guidance, Control, and Dynamics, 132-142.

Brunton, S., Fu, X., \& Kutz, J. (2013, Oct). Extremum-seeking control of a mode-locked laser. Quantum Electronics, IEEE Journal of , 49(10), 852-861. doi:

Calli, B., Caarls, W., Jonker, P., \& Wisse, M. (2012, Oct). Comparison of extremum seeking control algorithms for robotic applications. In Intelligent robots and systems (iros), 2012 ieee/rsj international conference on (p. 3195-3202). doi:

Camacho, E. F., \& Alba, C. B. (2013). Model predictive control. Springer Science \& Business Media.

Camacho, L. M., Dume, L., Zhang, J., Li, J.-d., Duke, M., Gomez, J., \& Gray, S. (2013). Advances in membrane distillation for water desalination and purification applications. Water, 5(1), 94-196. doi:

Choi, J.-Y., Krstić, M., Ariyur, K. B., \& Lee, J. S. (2002). Extremum seeking control for discretetime systems. Automatic Control, IEEE Transactions on, 47(2), 318-323.

Deschenes, J.-S., \& St-Onge, P. (2013, Dec). Achievable performances for basic perturbation-based extremum seeking control in wiener-hammerstein plants. In Decision and control (cdc), 2013 ieee 52nd annual conference on (p. 2991-2998). doi:

Drakunov, S., Ozguner, U., Dix, P., \& Ashrafi, B. (1995, Mar). Abs control using optimum search via sliding modes. Control Systems Technology, IEEE Transactions on, 3(1), 79-85. doi:

Eleiwi, F., Ghaffour, N., Alsaadi, A. S., Francis, L., \& Laleg-Kirati, T. M. (2016). Dynamic modeling and experimental validation for direct contact membrane distillation DCMD process. Desalination, 384, 1 - 11.

Eleiwi, F., \& Laleg-Kirati, T. (2015). Nonlinear lyapunov-based boundary control of distributed heat transfer mechanisms in membrane distillation plant. American Control Conference, $4964-4969$.

Eleiwi, F., \& Laleg-Kirati, T. M. (2014a). Dynamic modeling and optimization in membrane distillation system. Proceedings of the 19th IFAC World Congress, 3327-3332.

Eleiwi, F., \& Laleg-Kirati, T. M. (2014b). Membrane distillation process modeling: Dynamical approach. International Journal of Chemical, Nuclear, Metallurgical and Materials Engineering, 8(6), $479-484$.

Eleiwi, F., \& Laleg-Kirati, T. M. (2016). Nonlinear observer-based lyapunov boundary control of distributed heat transfer mechanisms for membrane distillation plant. Journal of Process Control.

Francis, L., Ghaffour, N., Alsaadi, A., Nunes, S., \& Amy, G. (2014). Performance evaluation of the dcmd desalination process under bench scale and large scale module operating conditions. Journal of Membrane Science, 455, 103-112.

Galeani, S., \& Teel, A. R. (2006). On a performance-robustness trade-off intrinsic to the natural anti-windup problem. Automatica, 42(11), 1849 - 1861.

Ghaffari, A., Krstić, M., \& Nesić, D. (2012). Multivariable newton-based extremum seeking. Automatica, 48(8), 1759 - 1767.

Godoy, B. I., Braslavsky, J. H., \& Agüero, J. C. (2008). A simulation study on model predictive control and extremum seeking control for heap bioleaching processes. Proc. IFAC World Congress.

Grimm, G., Teel, A. R., \& Zaccarian, L. (2004). Robust linear anti-windup synthesis for recovery of unconstrained performance. International Journal of Robust and Nonlinear Control, 14(1314), 1133-1168.

Guay, M., Dhaliwal, S., \& Dochain, D. (2013, June). A time-varying extremum-seeking control 
approach. In American control conference (acc), 2013 (p. 2643-2648). doi:

Kahne, S., \& Lee, E. (1967, June). Optimal control: An introduction to the theory and its applications. Automatic Control, IEEE Transactions on, 12(3), 345-347. doi:

Karam, A. M., \& Laleg-Kirati, T. M. (2015). Real time optimization of solar powered direct contact membrane distillation based on multivariable extremum seeking. IEEE Conference on Control Applications (CCA), Sydney, September 2015., 1 - 11.

Khayet, M. (2008). Advanced membrane technology and applications. In Advanced membrane technology and applications (pp. i-xxi). John Wiley \& Sons, Inc. doi:

Khayet, M., \& Matsuura, T. ((2011), 249 - 293). Chapter 10 - direct contact membrane distillation (M. K. Matsuura, Ed.). Amsterdam: Elsevier.

Krstić, M. (2000). Performance improvement and limitations in extremum seeking control, Systems $\&$ Control Letters, $39(5), 313$ - 326.

Krstić, M., \& Wang, H.-H. (2000). Stability of extremum seeking feedback for general nonlinear dynamic systems. Automatica, 36(4), 595-601.

Kwakernaak, H. (1972). Linear optimal control systems (R. Sivan, Ed.). New York, NY, USA: John Wiley \& Sons, Inc.

Lawson, K. W., \& Lloyd, D. R. (1997). Membrane distillation. Journal of Membrane Science, $124(1), 1-25$.

Lewis, F. L., Vrabie, D. L., \& Syrmos, V. L. (2012). Optimal control. John Wiley \& Sons, Inc. doi:

Li, P., Li, Y., \& Seem, J. (2009, June). Extremum seeking control for efficient and reliable operation of air-side economizers. In American control conference, 2009. acc '09. (p. 20-25). doi:

Li, X., Li, Y., Seem, J., \& Li, P. (2012, June). Extremum seeking control of cooling tower for self-optimizing efficient operation of chilled water systems. In American control conference (acc), 2012 (p. 3396-3401). doi:

Liu, S.-J., \& Krstić, M. (2012). Stochastic averaging and stochastic extremum seeking. Springer Science \& Business Media.

Moase, W., \& Manzie, C. (2012, July). Semi-global stability analysis of observer-based extremumseeking for hammerstein plants. Automatic Control, IEEE Transactions on, 57(7), 1685-1695. doi:

Moura, S., \& Chang, Y. (2010, June). Asymptotic convergence through lyapunov-based switching in extremum seeking with application to photovoltaic systems. , 3542-3548. doi:

Nesić, D. (2009, Aug). Extremum seeking control: Convergence analysis. In Control conference (ecc), 2009 european (p. 1702-1715).

Nesić, D., Mohammadi, A., \& Manzie, C. (2013, Feb). A framework for extremum seeking control of systems with parameter uncertainties. Automatic Control, IEEE Transactions on, 58(2), 435-448. doi:

Nesić, D., Tan, Y., Moase, W., \& Manzie, C. (2010, Dec). A unifying approach to extremum seeking: Adaptive schemes based on estimation of derivatives. In Decision and control (cdc), 2010 49th ieee conference on (p. 4625-4630). doi:

Raghavan, S., \& Hedrick, J. K. (1994). Observer design for a class of nonlinear systems. International Journal of Control, 59(2), 515-528. doi:

Sassano, M., Carnevale, D., Astolfi, A., \& Vergata, R. T. (2011). Extremum seeking-like observer for nonlinear systems. Proc. of the IFAC World Congress, 18.

Schofield, R., Fane, A., \& Fell, C. (1987). Heat and mass transfer in membrane distillation. Journal of Membrane Science, 33(3), 299 - 313.

Sharafi, J., Moase, W., Shekhar, R., \& Manzie, C. (2013, Dec). Fast model-based extremum seeking on hammerstein plants. In Decision and control (cdc), 2013 ieee 52nd annual conference on (p. 6226-6231). doi:

Simmons, G. (1995). Calculus with analytic geometry. McGraw-Hill Education.

Soudbakhsh, D., \& Annaswamy, A. (2013, June). Parallelized model predictive control. In American 
control conference (acc), 2013 (p. 1715-1720). doi:

Stankovic, M., \& Stipanovic, D. (2009, June). Stochastic extremum seeking with applications to mobile sensor networks. In American control conference, 2009. acc '09. (p. 5622-5627). doi:

Stankovic, M. S., \& Stipanovic, D. M. (2009). Discrete time extremum seeking by autonomous vehicles in a stochastic environment. In Decision and control, 2009 held jointly with the 2009 28th chinese control conference. cdc/ccc 2009. proceedings of the 48 th ieee conference on (pp. 4541-4546).

Tan, Y., Li, Y., \& Mareels, I. (2013, Sept). Extremum seeking for constrained inputs. Automatic Control, IEEE Transactions on, 58(9), 2405-2410. doi:

Tan, Y., Nesić, D., \& Mareels, I. (2006). On non-local stability properties of extremum seeking control. Automatica, 42(6), 889 - 903.

Tenne, Y., \& Goh, C. (2010). Computational intelligence in expensive optimization problems. Springer Berlin Heidelberg.

Thau, F. E. (1973). Observing the state of non-linear dynamic systems. International Journal of Control, 17(3), 471-479. doi:

Walcott, B., \& Zak, S. (1987, Feb). State observation of nonlinear uncertain dynamical systems. Automatic Control, IEEE Transactions on, 32(2), 166-170. doi:

Wang, H.-H., Krstić, M., \& Bastin, G. (1999). Optimizing bioreactors by extremum seeking. International Journal of Adaptive Control and Signal Processing, 13(8), 651-669.

Wang, H.-H., Yeung, S., \& Krstić, M. (2000). Experimental application of extremum seeking on an axial-flow compressor. IEEE Trans. Contr. Sys. Techn., 8(2), 300-309.

Ye, M., \& Hu, G. (2013, Dec). Extremum seeking under input constraint for systems with a time-varying extremum. In Decision and control (cdc), 2013 ieee 52nd annual conference on (p. 1708-1713). doi:

Zeilinger, M., Jones, C., \& Morari, M. (2011, Jûly). Real-time suboptimal model predictive control using a combination of explicit mpc and online optimization. Automatic Control, IEEE Transactions on, 56(7), 1524-1534. doi:

Zhang, C., \& Ordóñez, R. (2011). Extremum-seeking control and applications: a numerical optimization-based approach. Springer Seience \& Business Media.

Zhang, H., Chan, C., Cheung, K., \& Jin, H. (1998, Jun). Nonlinear observer design with unknown nonlinearity via b-spline network approach. In American control conference, 1998. proceedings of the 1998 (Vol. 4, p. 2339-2343 vol.4). doi: 